\title{
The Challenge of Launching Geology Samples off of Mars is Easily Underestimated, Due to Tempting Misconceptions
}

John Whitehead

$530-758-8115$

Consultant

john.whitehead@alumni.caltech.edu

A white paper submitted for the Decadal Survey on Planetary Science and Astrobiology

2020 June 21 (second of three by this author)

Revised with minor corrections, clarifications, and some additions

Resubmitted on 2020 September 11 


\section{Introduction}

This white paper is being offered with hopes that a broader community appreciation for the Mars ascent vehicle (MAV) challenge will help to ultimately bring Mars Sample Return (MSR) to fruition. The underlying concern is that the difficulty of developing a MAV for MSR has been widely underestimated over decades of time, for understandable reasons. Below are some potentially misleading notions that may seem logical at first glance, followed by explanatory information for each. The author has encountered many of these misconceptions among space enthusiasts, and among technical professionals, which motivated an overview in 2008 [1].

\section{Top Ten Potential Misconceptions about the MAV Challenge}

Misconception 1: We've landed on the moon and returned samples (U.S. Apollo and Soviet Luna), and we have also landed on Mars, so MSR should be a straightforward next step.

Explanation 1: This hopeful notion has been with us since Viking. While lunar descent and ascent need the same propulsive capability, Mars ascent is completely different from descent, so the capability to arrive and land on Mars says nothing about our ability to depart from Mars.

Misconception 2: The MAV needs a space propulsion system that will be designed and built on schedule and within budget, starting several years before departing from Earth, much like other propulsion systems for planetary spacecraft.

Explanation 2: Propulsive maneuvers done by planetary spacecraft are typically similar to what Earth satellites do (velocity changes and thrust-to-weight ratios), so planetary science has been lucky to benefit from highly reliable, proven technology and its broad base of expertise. In contrast, the MAV needs to be a miniature launch vehicle, with capability beyond the presently proven state of the art for its small size. The MAV project should be expected to include more trial-and-error development testing than any previous propulsion system for planetary science. The latter are only tested at the component level (e.g. tanks, valves, and engines are tested separately), but MAV work will include multiple flight tests at high altitudes over Earth.

Misconception 3: NASA has funded a series of engineering design studies for the MAV, starting more than 30 years ago, so there must be a good understanding of what needs to be done. Explanation 3: Studies were done on and off for so many years, because there is no easy answer [2-7]. MAV conceptual designs have not been detailed enough to be sure what will work, so no one really knows. The various studies have not been conclusive about the best way to build a MAV. Some studies were constrained by the premise that MSR would occur after a few more years, thereby ruling out official acknowledgement that MAV development might be a challenging long-term activity. In 1999 for example, MSR was going to be launched from Earth in 2003 and 2005 [8], so a long-term MAV effort was not envisioned. 
Misconception 4: The most important challenges for the MAV are surviving the journey to Mars, surviving the cold temperatures on Mars, and building a mechanism on the lander that lifts the MAV up to point it toward the sky in the correct direction.

Explanation 4: None of these things is especially easy, but it is a huge challenge, beyond what has ever been done, to build any rocket at all, small enough to send to Mars, that can reach Mars orbit from the surface. The required propulsive capability amounts to about half the speed of Earth orbit, so all the MAV components need to be unusually lightweight relative to the propellant mass. Most professional publications about potential MAV designs have not directly addressed this difficulty, and well-informed mass budgets are notably absent. There are limits to how thin metal can be manufactured, and then remain reliable when stressed. The miniaturization problem is partly related to the size of human hands.

Misconception 5: The MAV is just a little rocket, and many little rockets exist. Surely there is a solution in the world of small military missiles, for instance.

Explanation 5: Military missiles a little larger than a ton would not reach Mars orbit, while a MAV needs to be only a few hundred kilograms in order to fit on foreseeable Mars landers. Small military missiles go tens of miles, while a MAV would go thousands of miles over Earth.

Misconception 6: Developing a small MAV has got to be easier than developing a huge launch vehicle for Earth departure.

Explanation 6: Building a MAV should cost less, because facilities are much smaller and fewer people and raw materials are needed. However, the key difficulty, reducing the mass of rocket components relative to propellant mass, is more difficult for smaller vehicles. The largest Earth launch vehicles devote only one percent of their total launch mass to propellant containers, and one percent or so to engines, both of which are hard to do on a small scale. The SpaceX Falcon Heavy can deliver nearly 5 percent of its launch mass to low Earth orbit. The Rocket Lab Electron, roughly 100 times less massive, can orbit about 2 percent. Extrapolating toward smaller launch vehicles, the shape of the payload curve remains unknown, and at some size no payload is possible. The same principle applies to MAV scaling. It would not work to initially send a 10-kg MAV as a tech demo, as was done for rovers (Pathfinder Sojourner 1997).

Misconception 7: The MAV should be less complicated than a huge Mars rover with many science instruments, and less complicated than the multi-step systems for entry, descent, and landing (EDL). Therefore, developing a MAV should be easier.

Explanation 7: Hopefully the MAV can be less complex than a Mars rover or Mars EDL. However, complexity is not the only kind of difficulty. The physical reasons explained above present another kind of difficulty. One example of a physical difficulty would be asking for a rover that can drive up steep slopes, and never slide downhill or tip over.

Misconception 8: MAV technology can be developed and tested in a "breadboard" configuration to prove the propulsive functionality, then the excess weight can be carved out. 
Explanation 8: This general rule often works for electrical systems, e.g. the functionality of a heavy circuit board might be implemented on a microchip. For mechanical systems however, the physical implementation is more directly related to the functionality.

Misconception 9: Technology needed for a MAV will evolve from propulsion systems built for small commercial lunar landers.

Explanation 9: The surface gravity of Mars and the orbital velocity are more than twice those of the moon. While lunar landing and departure are possible using satellite propulsion methods, something very different will be needed for Mars ascent.

Misconception 10: A multi-stage solid propellant rocket seems like the obvious choice for a MAV. Solid rocket motors of the size needed for a MAV already exist, and those made for space (not missiles) have very low non-propellant mass (true). Some of the smallest Earth launch vehicles have been multi-stage solids (true, e.g. U.S. Scout and Pegasus, Japanese SS-520-5). Explanation 10: Solid rocket motors made for space maneuvers are mature and reliable, but typically they are used as part of a spacecraft that does the steering (either by spin stabilization or liquid propellant attitude control). Steering is especially challenging for a MAV, because smaller motors burn faster with excessive thrust forces [6], hence greater steering effort is required so the steering subsystem can be heavy. Short burn times result in high speeds and burnout while the MAV is still in the Mars atmosphere. High speeds come with aerodynamic drag losses, and the early burnout requires a second steering system to keep the MAV on course during the remaining time of complicated aerodynamic effects [7]. Spinning all stages can avoid the mass of guidance and steering components as done for the SS-520-5, but heavy ground equipment is needed for initial pointing and orbit precision may be compromised [9].

\section{Somewhat Anecdotal Indications of Limited Awareness Among Technical Professionals}

Misconception 11 (said by a professor lecturing about Mars meteorite analysis): The mass of Mars is only 11 percent of Earth's mass, so the lower gravity will make it easy to return samples. Explanation 11: The 11-percent mass ratio can be misleading. The minimum orbital velocity around a planet is proportional to the square root of the ratio, mass divided by radius, and the radius of Mars is only about half that of Earth. As a result, the speed for a low Mars orbit is 45 percent of the orbit speed over Earth. The same 0.45 ratio applies to escape velocities. Surface gravity, which determines the minimum thrust-to-weight ratio for launch, is proportional to mass divided by the square of radius. As a result, Mars gravity is 38 percent of Earth's.

Misconception 12 (said by more than one physicist): Increasing propulsive capability is simply a matter of using more propellant, relative to the payload. The mass of a rocket is dominated by the propellant, so we can neglect the rocket hardware mass for planning purposes.

Explanation 12: The statement amounts to asking for massless rocket components. Making them weigh less than the present state of the art, is the MAV engineering challenge. 
Misconception 13 (Caltech Magazine, fall 2019): Building a MAV is challenging because the components of a rocket don't do so well with extreme temperature changes, as on Mars. Explanation 13: While the statement is perfectly true, heaters and thermal insulation do exist. Ideal protection from the Mars environment would not solve the primary challenge to build a very small rocket that can accelerate all the way to Mars orbit.

Misconception 14 (Nature, Vol 448 p 978, 2007Aug30): MSR should be made affordable, by designing it to cost.

Explanation 14: Planetary science achievements that are successfully designed to fit predetermined budget limits, like Discovery missions or New Frontiers missions, benefit greatly from finding ways to accomplish new science goals using proven technology. In contrast, MSR needs new technology [10].

Misconception 15 (the author recalls being informed long ago that the following statement was spoken at a Mars science meeting): Building a MAV is blue collar work.

Explanation 15: The absence of a particular endeavor in universities might suggest that the subject matter is not mentally challenging. There are multiple reasons why rocket work in universities does not include making unusually lightweight components, as is needed for a MAV. Such efforts are too closely related to military missile work, which cannot be published openly, or shared freely among students and collaborators from multiple nations. Testing lightweight rocket parts is hazardous, considering that reactive substances need to be contained inside thin-walled components at high pressures and high temperatures. Third, related to the goals of education and open publishing, university engineering research in general leans toward science-like analysis, versus finalizing product design details. Educational institutions just happen to foster the incomplete notion that the intellectual part of rocket engineering is the details of what happens in thrust chambers, related to propellant chemistry and thermodynamics, propellant physical properties, intimate mixing of oxidizer and fuel, efficient smooth combustion, and nozzle shapes for flows to achieve high exhaust velocities. These topics are all very important, but more is needed.

Misconception 16 (said for decades, by many): Making propellants on Mars will make it easier to launch off of Mars, by avoiding the need to transport Mars-ascent propellants from Earth. Explanation 16: The worthy goal of in-situ propellant production (ISPP) has gradually advanced over decades. Finally, the MOXIE payload on Mars 2020 will demonstrate in-situ resource utilization (ISRU). ISRU and ISPP are important for long-term human exploration of Mars, assuming that the mass of materials produced can exceed the mass of production equipment sent from Earth to Mars. The latter is very unlikely to be practical on a small scale, for an initial few robotic MSR missions. Considering a MAV for MSR, the availability of rocket propellants does not change the need for a miniature launch vehicle to be made of components that are unusually lightweight relative to their size. 
Misconception 17 (recalled from an interview in an aerospace magazine in the late 1990's): The Mars samples are too valuable to risk on new propulsion technology.

Explanation 17: Something new and unusual is needed. See Explanations 1 through 10.

\section{Miscellaneous Potential MAV Misconceptions}

Misconception 18: The MAV team will meet requirements imposed by the team designing the sample return lander, which makes perfect sense because the MAV is a subsystem of the lander.

Explanation 18: Given that MAV development is a daunting task, it might make more sense to ask how the lander needs to be designed to accommodate whatever MAV that can be made to work. The sample return lander is a launch pad for a miniature launch vehicle, with support equipment for the MAV. For launches from Earth, launch pads and ground support equipment are designed to accommodate the needs of each launch vehicle.

Misconception 19: The MAV problem will be solved by finding propellants that can survive the cold thermal environment on Mars, including wide daily temperature cycles, while awaiting launch. Electrical heating could be provided by a radioisotope thermal generator (RTG), but the sample return lander should not be designed to need an RTG. The expense of RTGs should be reserved to power science instruments and environmental controls on large rovers.

Explanation 19: When developing a new propulsion system, relatively little progress can be made until the type of propellant is selected. However, selecting MAV propellants based on a single criterion might rule out a better overall solution. No one really knows to what extent it will be feasible for a MAV to wait on Mars with little or no environmental control. It might be more productive to ask which propellants will permit innovation toward unusually lightweight rocket components. Supporting the MAV with RTG power should not be ruled out.

Misconception 20: Propulsion research at universities will lead to MAV technology.

Explanation 20: Public research in the field of rocket propulsion is largely restricted to "propellants \& combustion," a blanket term for the chemistry and physical properties of rocket fuels and oxidizers, along with mixing and burning inside thrust chambers. This expertise is important, but experimental efforts in these fields rarely focus on making lightweight components that can be used for flight. Open research is significantly restricted by the fact that flight hardware meets regulatory definitions of military technology. See Explanation 15.

Misconception 21: MAV technology development efforts done decades ago are not relevant today, analogous to the obsolescence of old computer chips.

Explanation 21: Not much has changed in small-scale rocket technology, so old MAV ideas remain worthy of consideration. An example of slow change is that the sky crane landing engines, used for the Mars Science Laboratory (MSL, "Curiosity") and Mars 2020 ("Perseverance"), are derived from the ones that landed Viking on Mars in 1976. While special, 
these engines are not directly applicable to a MAV. Over recent decades, the one big change for satellite maneuvering is electric propulsion, but the thrust is way too low for launching.

Misconception 22: If an attempted technology development effort is not successful after a few years, it will be passed by and overcome by other developments.

Explanation 22: This statement is often true in the computer world, but Explanation 21 applies.

Misconception 23: If the MAV is so much better than existing military missiles, then MAV technology will lead to new weaponry, or maybe there is advanced missile technology in the pipeline that will turn out to solve the MAV problem.

Explanation 23: The speed and distance of missiles is limited, partly because they must be structurally robust to withstand field handling in combat situations, and partly because they are designed for quantity production. A MAV will be structurally delicate by comparison, in order to achieve a sufficiently low mass sum for all the components. The MAV is going to be unique and specialized in multiple ways, thus unlikely to be practical for military use.

Misconception 24: Modern design software is a huge time saver for reducing schedule risk. Explanation 24: Three-dimensional computer aided design (3D CAD) has greatly improved some aspects of engineering development, but not all. One concern to be aware of is that 3D CAD renderings can blur the distinction between engineering progress and conceptual designs.

Misconception 25: If MSR is postponed again, the necessary technology will eventually appear on the scene for some other reason.

Explanation 25: There are healthy "technology pipelines" for many items and methods that can be useful for building planetary spacecraft. For example, many research efforts continue to improve imaging sensors. However, as illustrated in Figure 1, no commercial markets, or other government customers, are driving investment in miniature launch vehicles as small as the MAV needs to be. One possibility is that there might someday be very
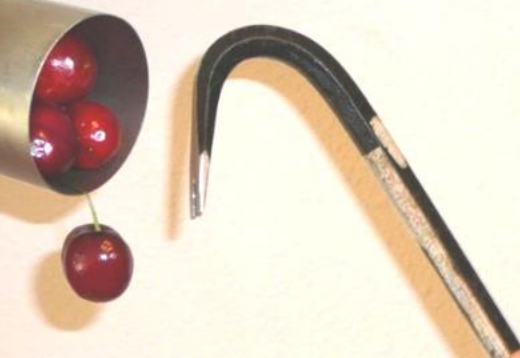
small Earth launch vehicles, dedicated for launching individual cubesats. The upper stages of such hypothetical tiny launch vehicles might be similar to a MAV, but many details would most likely need to differ.

Figure 1. Not every problem can be solved by leveraging lowhanging fruit as it emerges from the technology pipeline. 


\section{$\underline{\text { References cited }}$}

1. Whitehead, J.C., "Defining the Mars Ascent Problem for Sample Return," American Institute of Aeronautics and Astronautics, AIAA-2008-7768, Space 2008, San Diego, CA, September 2008. https://arc.aiaa.org/doi/abs/10.2514/6.2008-7768 https://arc.aiaa.org/doi/10.2514/6.2008-7768

2. Lance, N., "Mars Rover Sample Return Ascent, Rendezvous, and Return to Earth," American Institute of Aeronautics and Astronautics, AIAA-1989-0424, 27th Aerospace Sciences Meeting, Reno, NV, January 1989. https://arc.aiaa.org/doi/10.2514/6.1989-424

3. Guernsey, C., et al, "Evaluation of Some Candidate Propulsion Technologies for Mars Ascent," American Institute of Aeronautics and Astronautics, AIAA-1998-0651, 36th Aerospace Sciences Meeting, Reno, NV, January 1998. https://arc.aiaa.org/doi/10.2514/6.1998-651

4. Stephenson, D., "Mars Ascent Vehicle--Concept Development," American Institute of Aeronautics and Astronautics, AIAA-2002-4318, Joint Propulsion Conference, July 2002. https://arc.aiaa.org/doi/abs/10.2514/6.2002-4318 https://arc.aiaa.org/doi/10.2514/6.2002-4318

5. Dankanich, J.W. and Klein, E., "Mars Ascent Vehicle Development Status," 2012 IEEE Aerospace Conference, Big Sky, MT, March 2012. https://ieeexplore.ieee.org/document/6187295

6. Shotwell, R., et al, "Drivers, Developments, and Options Under Consideration for a Mars Ascent Vehicle," 2016 IEEE Aerospace Conference, Big Sky, MT, March 2016. https://ieeexplore.ieee.org/document/7500822

7. McCollum, L.T., et al, "Development Concepts for Mars Ascent Vehicle (MAV) Solid and Hybrid Vehicle Systems," 2019 IEEE Aerospace Conference, Big Sky, MT, March 2019. https://ieeexplore.iee.org/abstract/document/8741965

8. Price, H., et al, "Mars Sample Return Spacecraft Systems Architecture," 2000 IEEE Aerospace Conference, Big Sky, MT, March 2000. https://ieeexplore.ieee.org/document/879302

9. Inatani, Y., and Ohtsuka, H., "SS-520 Nano Satellite Launcher and its Flight Result," 32nd Annual Small Satellite Conference, SSC18-IX-03, August 2018. https://digitalcommons.usu.edu/smallsat/2018/all2018/308/

10. Whitehead, J.C., "Mars needs technology designed for sample return," Nature, Vol. 449, p 972, 25 October 2007. https://www.nature.com/articles/449972c 